\title{
CAMPUS UNIVERSITARIO PARA EL DESARROLLO LOCAL Y REGIONAL: PLAN MAESTRO PARA EL FUTURO DE LA UNIVERSIDAD DE CONCEPCIÓN EN LA AGRO-REGIÓN DEL ÑUBLE
}

University campus for local and regional development: Master plan for the future of the University of Concepción in the agro-region of Nuble

\section{MONTSERRAT DELPINO CHAMY* \\ PEDRO ORELLANA AGÜERO** \\ FRANCO ROMERO***}

RESUMEN: Los campus universitarios operan como instrumentos de relación entre la universidad y su medio urbano. Su localización en ciudades intermedias resulta clave para dinamizar el desarrollo local. El siguiente artículo aborda las potencialidades del desarrollo futuro del campus Chillán de la Universidad de Concepción, Chile, localizado en una región de alto valor agrícola. Con 97 hectáreas de superficie funciona como el "laboratorio vivo" más grande del país. Sin embargo, se encuentra inmerso en una ciudad que ha ido perdiendo su suelo agrícola en favor del crecimiento horizontal de baja densidad, con la consecuente fragmentación de su estructura social y urbana. En este contexto, tanto por su localización, prestigio y extensión, el campus Chillán ofrece una oportunidad única para promover un desarrollo local más sustentable, acorde a la vocación agrícola de su territorio. La metodología del estudio es cuantitativa y documental, utilizando encuestas de percepción y la construcción de análisis de amenazas y oportunidades para proponer planes maestros. Para lo anterior, se desarrolla un análisis de los sistemas urbanos de Chillán en la interfaz campus-ciudad y se presentan 4 propuestas de master plan desarrolladas por el equipo de estudiantes de Proyecto IV, en la Facultad de Arquitectura, Urbanismo y Geografía. Este trabajo se enmarca en la conmemoración de los 100 años de la Universidad de Concepción y ofrece lineamientos para pensar el futuro hacia el próximo bicentenario, mediante estrategias que permitan estimular la economía local, la innovación y el surgimiento de nuevos espacios y servicios para la comunidad.

PALABRAS CLAVES: Campus universitario, ciudad agrícola, desarrollo regional, master plan, sostenibilidad urbana.

\footnotetext{
* Doctora (c) Facultad de Arquitectura, Urbanismo y Geografía, Universidad de Concepción, Concepción, Chile. mdelpino@udec.cl ORCID: https://orcid.org/0000-000 1-8607-6097

**Arqto. Facultad de Arquitectura, Urbanismo y Geografía, Universidad de Concepción, Concepción, Chile. pedorell@udec.cl ORCID: https://orcid.org/0000-0002-7396-025 1

*** Arquitecto egresado de la Facultad de Arquitectura, Urbanismo y Geografía, Universidad de Concepción, Concepción, Chile.
} 
ABSTRACT: University campuses operate as instruments of connection between universities and their urban environments. Their location in intermediate cities is key to stimulate local development. The following article addresses the potential of the future development of the Chillán campus of the Universidad de Concepción, Chile, located in a region of high agricultural value. With 97 hectares, it functions as the largest "living laboratory" in the country. However, it is immersed in a city that has been losing its agricultural land in favor of low-density horizontal growth, with the consequent fragmentation of its social and urban structure. In this context, due to its location, prestige and extension, the Chillán campus offers a unique opportunity to promote more sustainable local development, in keeping with the agricultural vocation of its territory. With this in mind, the urban systems of Chillán at the campus-city interface are analyzed, and 4 master plan proposals developed by the team of students of Project IV, at the Faculty of Architecture, Urbanism and Geography of the University of Concepción are presented. This work is part of the commemoration of the 100 years of the University of Concepción, and offers guidelines for thinking about the future in expectation of the next bicentennial, through strategies that stimulation of the local economy, innovation and the emergence of new spaces and services for the community.

KEY WORDS: University campus, agricultural city, regional development, master plan, urban sustainability.

\section{INTRODUCCIÓN}

El presente artículo se enmarca en la celebración del centenario de la Universidad de Concepción (UdeCly respondea una invitación del rector Carlos Saavedra Rubilar para pensar la universidad y sus campus por los próximos 100 años. Este desafío es internalizado por la Facultad de Arquitectura, Urbanismo y Geografía, confiando su realización al trabajo conjunto entre estudiantes.

El caso del Campus Chillán es abordado por el Taller de Arquitectura "Proyecto IV", cuyo equipo docente fue conformado por los autores de esta publicación El trabajo se planificó conformando encargos interdisciplinares entre futuros Agrónomos del campus Chillan y futuros Arquitectos y Geógrafos del campus Concepción. Este enfoque interdisciplinar fue compartido con los profesores JuanAlberto Barrera Berrocal subdirector y Pedro Rojas García director del Campus Chillan, quienes brindaron una clase magistral para el estudiantado, así como toda la colaboración y material de apoyo al equipo docente.

El trabajo de todo el semestre es sistematizado en un libro y en este artículo, que se estructura siguiendo la metodología de taller. Inicia con una breve reseña histórica que rescata los destacados orígenes del campus y continúa con un análisis urbano que analiza la relación campus-ciudad y evalúa los sistemas de Movilidad, Equipamientos, Áreas verdes y Percepción ciudadana. Posteriormente, presenta cuatro propuestas de plan maestro desarrolladas por el estudiantado en base al análisis urbano, dejando en evidencia el rol estratégico del Campus Chillán, tanto para el desarrollo regional como nacional. Finalmente, el documento cierra con un resumen de las principales potencialidades identificadas para el desarrollo de un campus universitario sustentable, que permita celebrar con júbilo el próximo bicentenario de la Universidad de Concepción. 


\section{MARCO TEÓRICO}

\section{Campus Universitario para una ciudad de vocación agrícola}

Los campus universitarios surgen en Chile a principios del S.XX como espacios urbanos que concentran distintas edificaciones dedicadas a la educación superior, articuladas por extensas áreas verdes (Coss Lanz, 2010). El campus de la Universidad de Concepción es el pionero a nivel nacional. Su construcción, iniciada en 1921 en la ciudad de Concepción, posteriormente la regula el plan regulador de Karl Brunner (1931), seguido de Emilio Duhart (1957). Su planificación rescata los principios del movimiento moderno y busca articularse con el tejido urbano, entregando espacios comunes y colectivos a la ciudad (Fuentes, 2007).

En 1954, producto de una colaboración entre la Universidad de Concepción, el Ministerio de Agricultura de Chile y el Instituto de Asuntos Interamericanos de Estados Unidos, se funda en la ciudad de Chillán la Facultad de Agronomía de la Universidad de Concepción, sobre un predio de 97 hectáreas. Esta decisión se enmarcaen las líneas estratégicas del Plan Chillán.

Su finalidad apunta a reactivar la rezagada economía local mediante el desarrollo científico y tecnológico para el aprovechamiento del alto valor agropecuario de la localidad (Soto y Escobar, 2016).

La fundación del Campus de la Universidad de Concepción permitió renovar el rol agrícola de la ciudad potenciado por la llegada del ferrocarril en 1836, el cual permitió distribuir la producción agrícola de la zona hacia los puertos del país. Simultáneamente permitió recuperar la tradición formativa instalada por la Escuela Práctica de Agricultura de Chillán de 1881 (Briones, Hernández y Mancilla, 2019). El cuerpo docente de la nueva Facultad de Agronomía se conformó con un prestigioso equipo de profesionales del agro, formados en Europa y Estados Unidos. A ellos se asociaron laboratorios, equipos, bibliotecas especializadas e instalaciones técnicas, que permitieron a Chillán reposicionarse como un polo agrícola de liderazgo a nivel nacional (Universidad de Concepción, 2020).

El éxito del campus Chillán hizo necesaria la creación de otras carreras universitarias afines a las actividades agropecuarias y al desarrollo regional. Se funda así Medicina Veterinaria en 1973, Ingeniería Forestal en 1977, Ingeniería Civil Agrícola en 1988, Ingeniería Agroindustrial en 2005, Ingeniería Comercial en 2005, Derecho en 2006, Ingeniería en Alimentos en 2009, Ingeniería Ambiental en 2010 y, finalmente, en el año 2013 la Carrera de Enfermería (Universidad de Concepción, 2020) (ver figura 1).

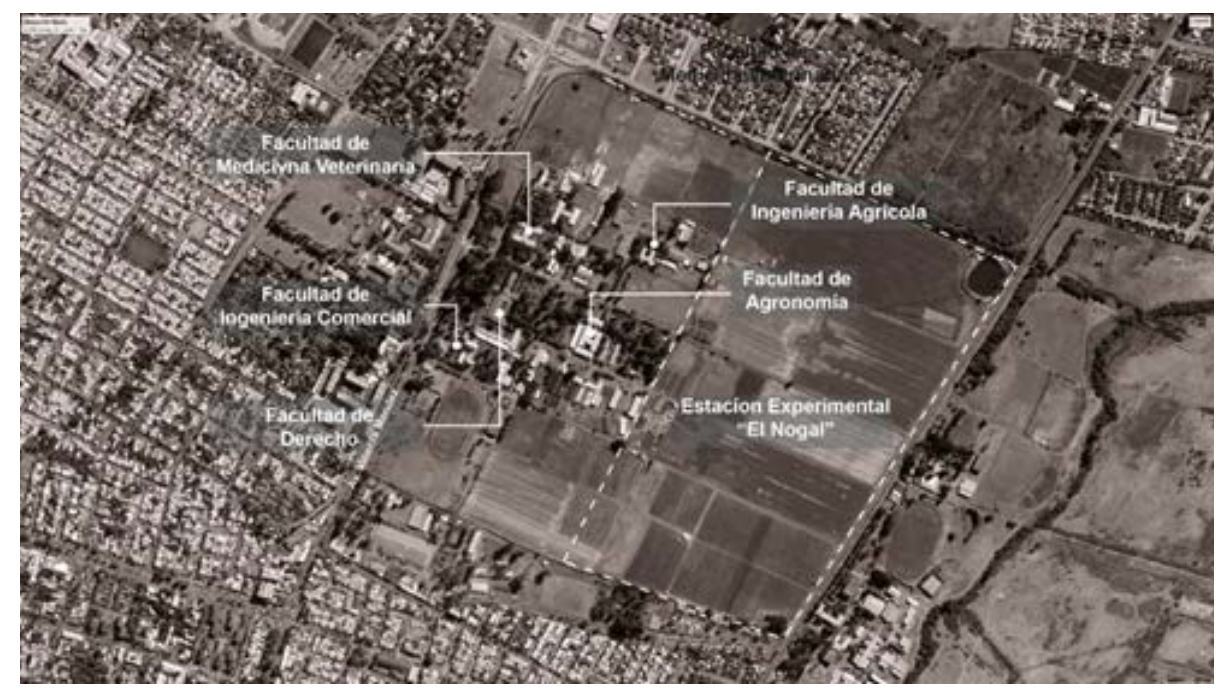

Figura 1. Distribución Campus Chillán Universidad de Concepción, Chillán, Chile.

Fuente: Elaboración propia. 
A la fecha el campus consta de más de $30.000 \mathrm{~m} 2$ construidos, distribuidos entre árboles y jardines que confieren a esta sede una imagen de antiguo parque. Las edificaciones se concentran en la zona poniente, manteniendo liberados más de 50 hectáreas asociadas a la zona oriente, donde se emplaza la "Estación Experimental El Nogal". Esta estación posee pequeñas edificaciones de valor patrimonial que apoyan la investigación sobre extensas zonas de cultivos, convirtiendo este centro en el único "laboratorio vivo" del país (Soto y Escobar, 2016).
El crecimiento urbano y el consumo de la base agrícola productiva

A medida que el Campus Chillán se desarrollaba también lo hacía su ciudad, pasando de una superficie de 830 hectáreas en 1940, a 3220 hectáreas en el 2014 (Soto y Escobar, 2016). Estas dinámicas de crecimiento han respondido a procesos de expansión horizontal, las cuales han triplicado la superficie de la mancha urbana en 70 años (ver figura 2), reduciendo significativamente la cantidad de suelos agrícolas de alta calidad en torno a la ciudad y consumiendo así la base del dinamismo económico de la región.
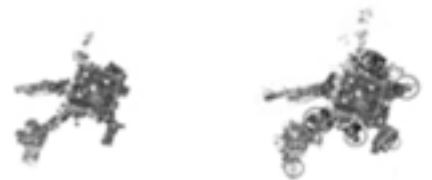

1940 830,99 ha
1960 $1.045,59$ ha

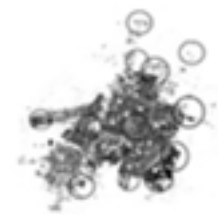

$1.518,89$ ha

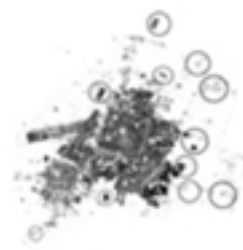

$2.397,01$ ha

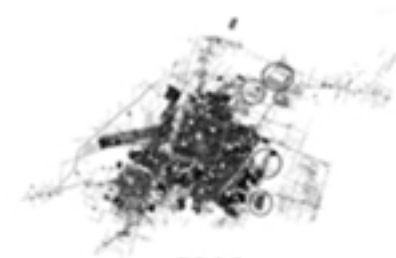

2014

$3.220,23$ ha

Figura 2. Crecimiento urbano de Chillán, Chile. Fuente: Soto \& Escobar (2016).

La ciudad perdió 854 hectáreas de suelo agrícola entre los años 1980 y 2000, cifra que aumenta a más de mil hectáreas de suelo agrícola urbanizado entre los años 2000 y 2014 (Soto y Escobar, 2016). A esto se le suma una fuerte fragmentación socioeconómica, localizando a poblaciones de bajos ingresos en las zonas suroriente y a familias más acomodadas en el sector nororiente, mediante el surgimiento de condominios privados y parcelas de agrado (Anabalón, 2006).

En el año 2018 se funda la nueva Región de Nuble y Chillán se promulga como su capital. Con más de 150.000 habitantes, esta ciudad pasa a transformarse en el nuevo centro de desarrollo administrativo de la Región y es llamada a desempeñarse como una ciudad intermedia, capaz de articular pequeñas localidades circundantes con los grandes centros urbanos a nivel nacional y mundial (Llop, Iglesias, Vargas y Blanc, 2019).

En este contexto, se evidencia como tanto la ciudad como el campus Chillán de la Universidad de Concepción cumplen un rol fundamental en el desarrollo futuro de la nueva Región de Nuble. El desafío radica en buscar formas alternativas de planificación que permitan rescatar los principios fundacionales del campus y la universidad, preservar el valor agrícola de su suelo y simultáneamente dinamizar la actividad investigativa, económica y la vinculación con el medio urbano, colaborando en la construcción de un futuro más sustentable para la región y el país. 


\section{METODOLOGÍA}

El estudio presenta un paradigma de investigación mixto. Inicialmente se utiliza un paradigma cuantitativo de corte descriptivo y transversal para el fenómeno de estudio. Complementado por un análisis de fuentes a través de documentos de la ciudad de Chillán y el Campus Universitario.

Este enfoque metodológico es utilizado por el equipo de 3 er año de arquitectura en la Universidad de Concepción para desarrollar un análisis de los sistemas urbanos de la ciudad y su relación con el campus universitario de Chillán. Los sistemas urbanos analizados están compuestos por la movilidad, equipamientos y áreas verdes y responden a un enfoque simplificado de la metodología de estudio utilizada por la Agencia de Ecología Urbana de Barcelona (Rueda, 2012).

La movilidad puede ser comprendida a través de la relación distancia - tiempo, siendo caracterizada por los procesos y formas de desplazamiento entre localizaciones de los sujetos (Lizárraga, 2006). El equipamiento representa la agrupación de edificios y espacios de uso público en donde se accede a servicios sociales como la salud, recreación, educación o cultura (Alcantará, 2010). Y las áreas verdes representan superficies permeables, que ofrecen servicios ecosistémicos y ayudan a la cohesión social en las ciudades (Cáceres y Ahumada, 20181 .

Para este análisis, la recolección de la información se logró a través de documentos oficiales y archivos fotográficos $y$, para conocer la percepción de las personas y estudiantes respecto al campus y la ciudad, se realizó un levantamiento de encuestas y un mapeo participativo donde participaron 81 miembros de la comunidad UdeC y 35 residentes de la ciudad de Chillán, 116 personas en total.
Luego, la información fue procesada a través de fases: a) análisis de oportunidades y amenazas para la ciudad y el campus; b) descripción univariada de los resultados de las encuestas de percepción; y c) construcción de propuestas de planes maestros para la relación campus - ciudad.

\section{RESULTADOS \\ Oportunidades y amenazas de la relación campus- ciudad.}

El campus constituye la expresión física de la universidad y opera como instrumento de relación entre ésta y el medio urbano (Behm Rosas, 1969 citado en Fuentes, 2007). De hecho, los campus universitarios son valiosos agentes de desarrollo local, estimulando la demanda de bienes y servicios, desarrollando capital humano, mejorando la productividad de negocios aledaños y ofreciendo nuevos espacios y servicios para la comunidad (Rossi y Goglio, 2020).

Reconociendolos beneficiossocioeconómicos de la interfaz campus-ciudad, este capítulo identifica las principales oportunidades y amenazas en términos de principios de sostenibilidad urbana, asociada al análisis de los principales sistemas urbanos de la ciudad de Chillán: movilidad, equipamientos y áreas verdes (Rueda, De Caseres, Cuchí, y Braul, 20121.

\section{Sistema de Movilidad, viabilidad y flujos}

La ciudad cuenta con una población de 156.537 habitantes, de los cuales el $88 \%$ se concentra en la ciudad y un $12 \%$ en las zonas rurales (Anabalón, 2006). Chillán es una ciudad intermedia de segunda jerarquía dentro del sistema de vertebración vial del territorio (García-Huidobro y Maragaño, 2010). Su ubicación geográfica, en un valle interior entre dos sistemas cordilleranos, garantiza una fácil accesibilidad desde todas direcciones. Estos factores motivan una buena conectividad a una meso escala. 


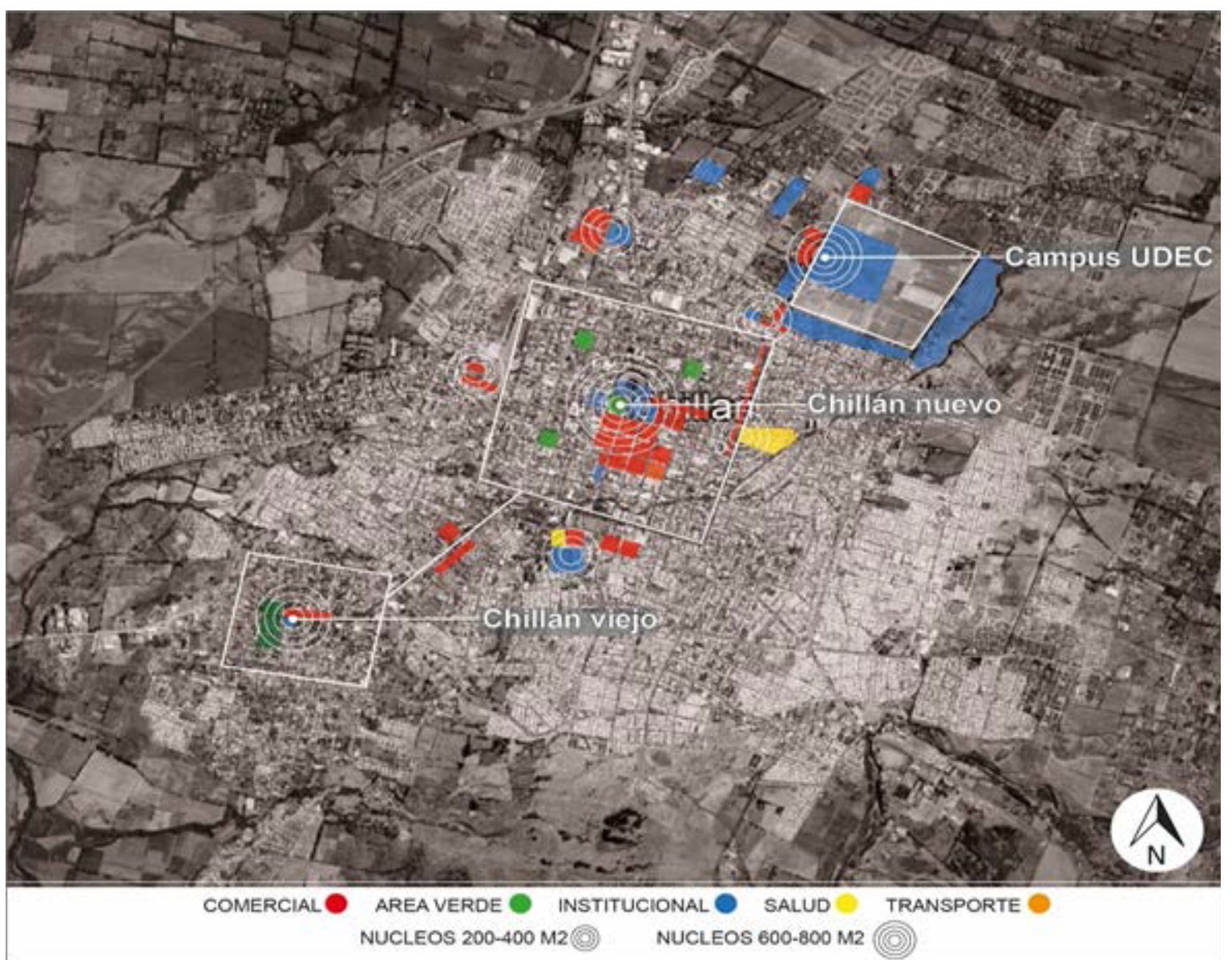

Figura 3. - Relación campus-Chillán nuevo -Chillán viejo y equipamiento. Fuente: Elaboración propia.

Su estructura urbana está conformada por dos centros, algo poco usual. El primer centro, Chillán Viejo, cuenta con el núcleo cívico de la ciudad en donde opera gran parte del aparato funcional y comercial. El segundo centro, Chillán Nuevo, posee un rol más residencial (ver figura 3).

En su área urbana consolidada, Chillán Nuevo posee dimensiones cercanas a los 1,5 kilómetros de radio. Considerando velocidades promedio de desplazamiento peatonal, se puede catalogar como una ciudad caminable, que no llevaría más de 30 minutos para atravesarla.

La universidad posee una comunidad aproximada de 2.800 personas, lo cual significa un 1,7\% de la población total de Chillán que se moviliza hacia ésta y se encuentra a 2400 metros del centro fundacional de Chillán Nuevo (30 mins caminando), distancia análoga a la que el Campus Concepción de la UdeC tiene con el centro fundacional de Concepción (22 mins caminando). A pesar de su cercanía, la locomoción pública cumple un rol fundamental, siendo la forma de movilidad más utilizada. En particular se cuenta con cinco líneas entre autobuses y taxis que conectan los núcleos principales de Chillán con el campus UdeC, esto se debe a la carencia de comercio y centralidades entre el centro fundacional y el campus, lo que desincentiva la caminabilidad.

Por tanto, a pesar de que Chillán cuenta con posibilidades claras de responder a principios de movilidad más sostenible (caminabilidad, desplazamientos en bicicleta), la carencia de comercio y centralidades barriales lleva a las personas a optar por medios de movilización 
como el automóvil y transporte colectivo, lo que desemboca en problemas viales de atochamientos y contaminación.

\section{Sistema de Equipamientos}

El asesor urbanista de la Municipalidad de Chillán ha caracterizado esta localidad como un centro urbano que contiene tres ciudades en una (Anabalón, 2006). La primera, correspondiente a los cascos históricos; la segunda, "la ciudad de los pobres", en la zona sur-oriente y, la tercera, "la ciudad de las oportunidades", en la zona nor-oriente. En medio de estas dos últimas se emplaza el campus Chillán de la Universidad de Concepción (ver figura 4).

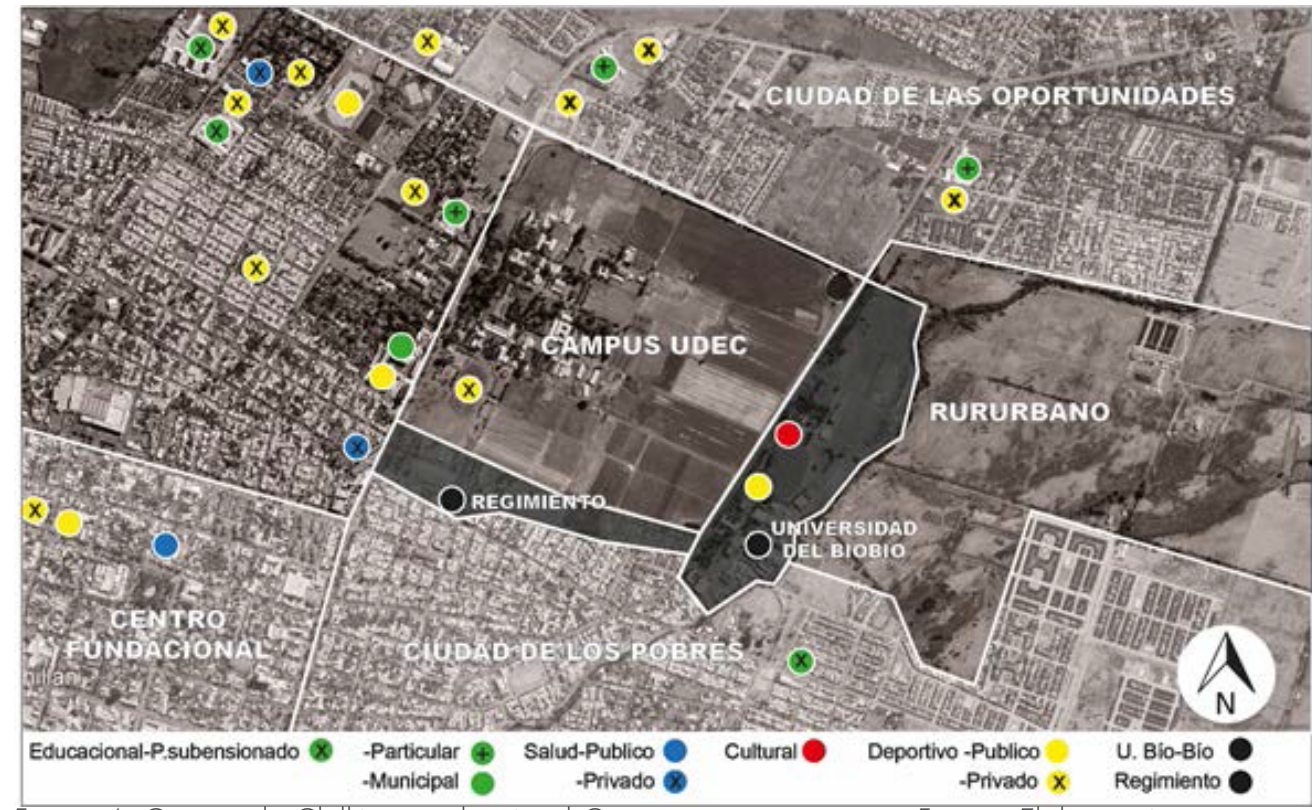

Figura 4. Centros de Chillán en relación al Campus y equipamiento. Fuente: Elaboración propia.

El entorno urbano de la Universidad de Concepción destaca por tener mucho potencial en equipamiento educacional y deportivo de calidad, hacia su zona norte y poniente. No obstante, carece de equipamientos culturales en este sector. Complementariamente la zona sur presenta más carencias. Se observa además la presencia de un antiguo regimiento de infantería, emplazado entre la Universidad de Concepción y los barrios residenciales hacia el sur, lo que da discontinuidad al tejido urbano. Finalmente, hacia el oriente se localiza la Estación Experimental El Nogal de la UdeC; la Universidad del Bío Bío, Campus Fernando May y el estero Las Toscas, tres elementos que contribuyen al carácter rural de esta zona.

\section{Sistema de Áreas Verdes}

A pesar de ser una ciudad de vocación agrícola, las áreas verdes en Chillán presentan graves carencias. En 1996, el total de áreas verdes por habitante era de 1,66 m2 (Gonzalez y Olave, 1996). El Plan de Desarrollo Comunal (PLADECO) del año 2019 declara un aumento a 2,3 m2/hab, considerando sólo aquellas áreas verdes de administración pública. Estas cifras son muy inferiores a los 9 u 11 m2 de áreas verdes/hab recomendados por organismos internacionales como la Organización Mundial de la Salud (OMS) (Gonzalez y Olave, 1996).

En medio de esta escasez de infraestructura verde algunos de los espacios significativos de la ciudad son: Parque de los Artistas, Plaza Santo Domingo, Plaza Victoria, Plaza San Francisco y áreas verdes asociadas a vialidad: Avenida Brasil, Avenida Ecuador, Avenida Collin y Avenida Argentina (ver figura 5). 


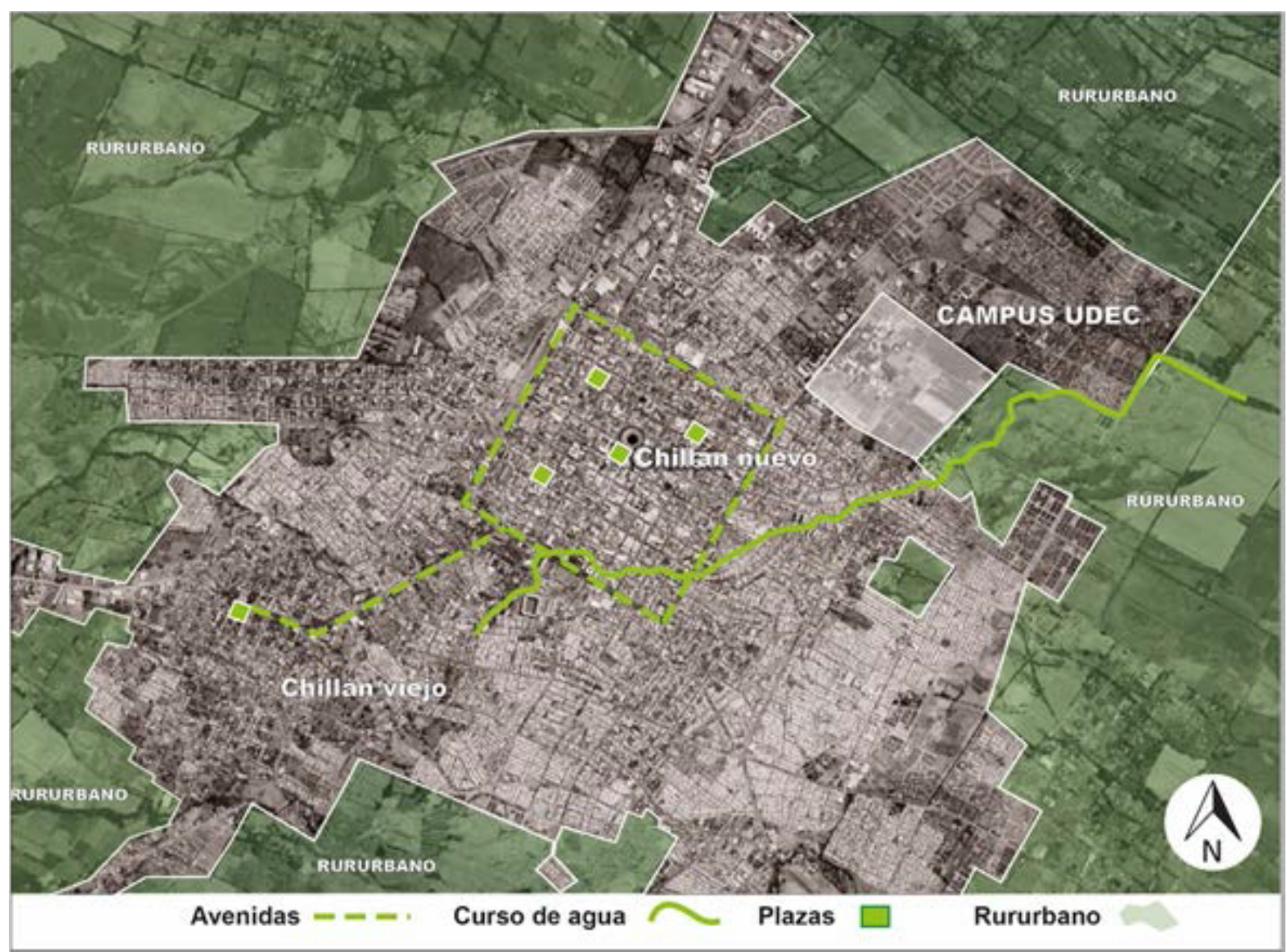

Figura 5. Áreas verdes de la ciudad de Chillán, Chile. Fuente: Elaboración propia.

Si bien el campus Chillán de la Universidad de Concepción es cerrado, en su interior contiene 87 hectáreas de áreas verdes y espacios de cultivo. Si el campus habilitara su infraestructura verde para el disfrute de la población local Chillán aumentaría de 2,3 a 8 m2 de áreas verdes por habitante.

Percepción sobre el campus universitario y la ciudad de Chillán

Los edificios más frecuentados son los que concentran mayor cantidad de servicios y mejor accesibilidad (Edificio Central, Biblioteca, Casinol. En general la percepción es positiva respecto a la seguridad, aunque se identifican zonas de riesgo; y favorable en cuanto a la existencia de canchas, espacios comunes y quinchos. Se critica la calidad de los edificios y las aulas. Lo más valorado son las áreas verdes y la relación de cercanía con docentes y sentido de comunidad en general (ver figura 6).

Entre los lugares más importantes de Chillán se mencionaron la Plaza de Armas, El complejo deportivo Quilimapu, el Mercado y la Plaza la Victoria. Ninguna de las personas encuestadas identificó el Campus UdeC como un lugar importante dentro de la ciudad. De entre los encuestados, el $26 \%$ nunca ha visitado el campus y el $61 \%$ no suele visitarlo. Sin embargo, el 74\% indicó que iría a pasear al campus con gusto o mucho agrado y el 100\% indicó que lo visitaría si adoptara rol de parque. 


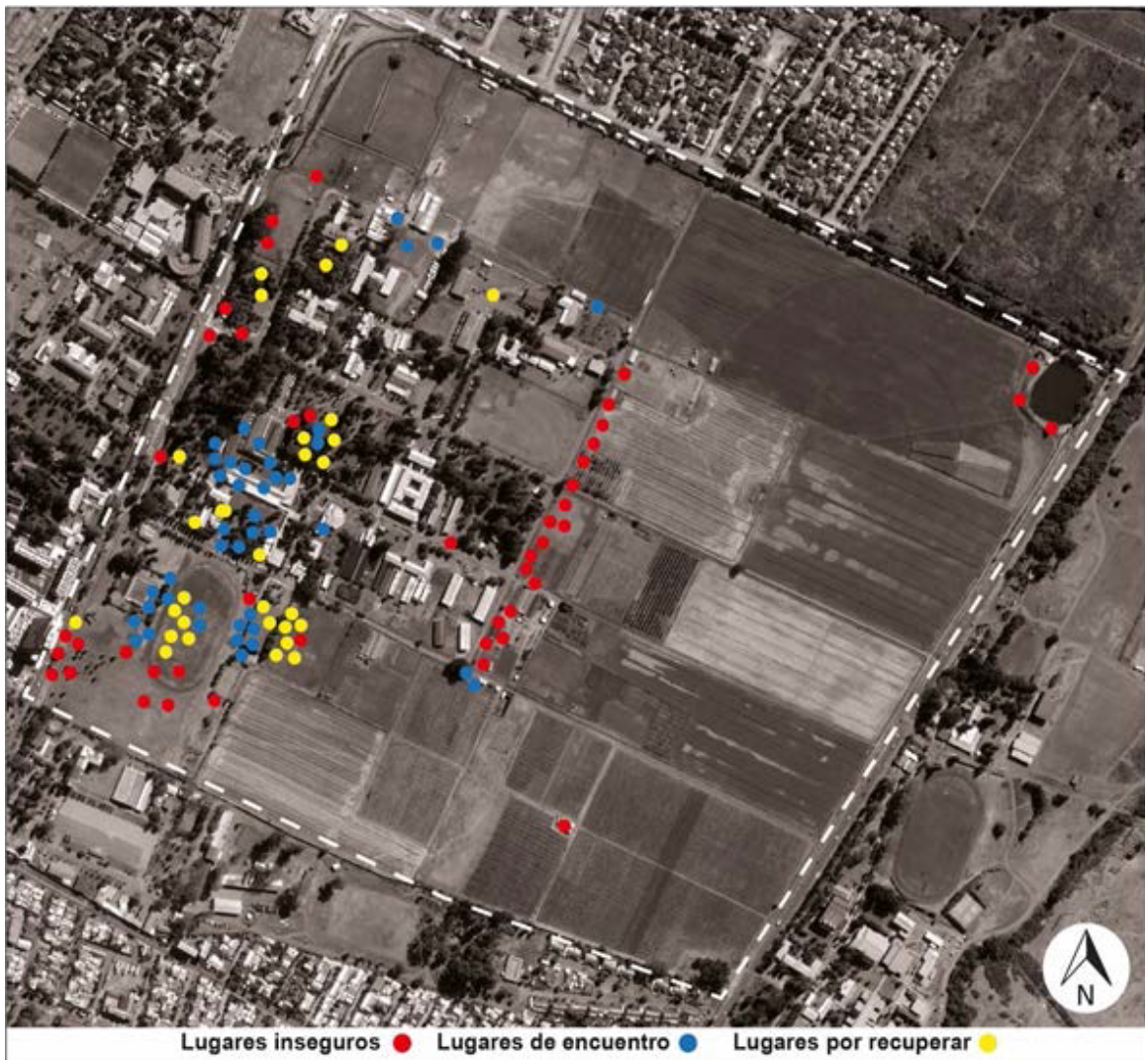

Figura 6. Percepción de la comunidad UdeC respecto al Campus Chillán, Chillán, Chile. Fuente: Elaboración propia.

Alternativas para el desarrollo futuro de un campus sostenible para Chillán

Posterior al análisis de la relación campusciudad, el equipo de estudiantes del taller Proyecto 4 trabaió cuatro imágenes de planes maestros, que nacen de la oportunidad de aportar al desarrollo de la naciente región de Nuble, así como a la ciudad capital de Chillan y el barrio donde está emplazado el campus universitario. Así, las ideas planteadas abordar las relaciones desde estas tres escalas, teniendo por centro la relación entre la comunidad académica y la población local (Dalton, Hajrasouliha, \& Riggs, 20181.

Plan maestro "Conexión entre plazas"
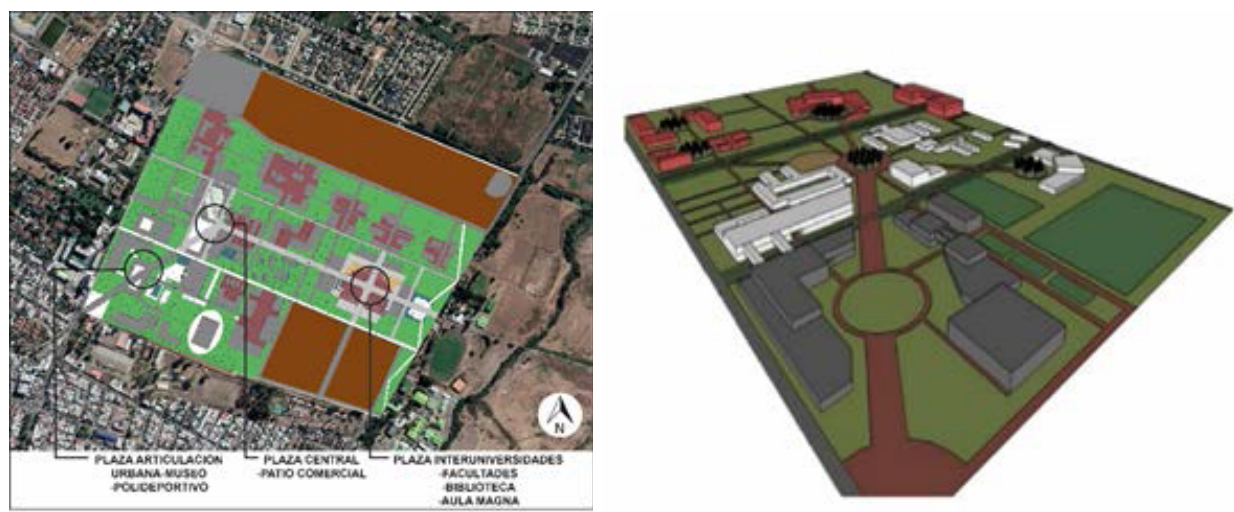

Figura 7. Masterplan Conexión entre plazas. Fuente: Felipe Quezada, Javiera Solar, Sebastián Rivera y Constanza Riquelme. 
Este máster plan propone unir el recintouniversitario con la ciudad consolidando un eje urbano que configure una secuencia de plazas dentro de la trama urbana de Chillan, y que conduciría peatonalmente al terreno de la universidad. Trayecto que remataria en la gran plaza de Agronomía, como signo del desarrollo que esta disciplina debe aportar para consolidar la región de Nuble.(ver figura 7).

A la escala de su barrio plantea, desde el corazón, conectar peatonalmente al norte con las nuevas áreas residenciales que están consolidando el crecimiento de la ciudad. Y de igual forma hacia la localización del campus de la Universidad del Bio-Bio, es decir integrarse con una red peatonal al entorno inmediato y facilitar el paso de peatones. Ambos ejes peatonales de integración al entorno y de paso, resguardaran el suelo destinado a la investigación agrícola y trabajo de campo con animales, demarcando una zona más privada y netamente universitaria.

\section{Plan maestro "Parque histórico universitario"}
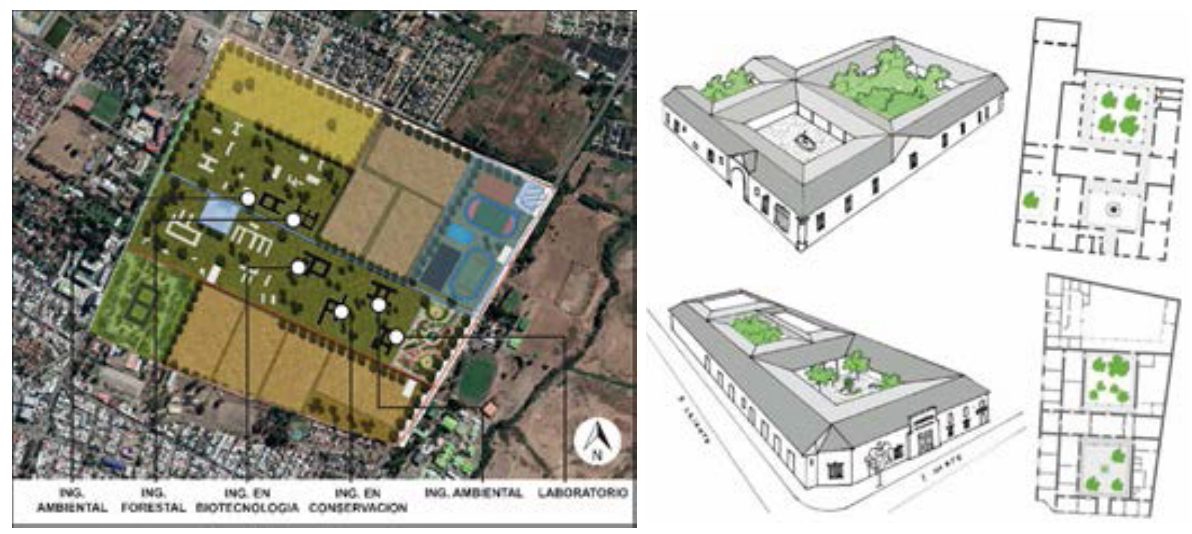

Figura 8. Masterplan Parque histórico universitario. Fuente: Catiray Henríquez, Daniela Jarpa y Esteban Rodríguez

Este plan maestro propone una zonificación y morfología arquitectónica relacionada con la historia de figuras ilustres que forjaron la Región de Nuble. Simultáneamente rescata los principios de diseño ligados a las viviendas típicas del campo, incorporando corredores periféricos y patios interiores. Esta estrategia permite articularse con la historia, y aprovechar los beneficios de eficiencia energética asociados a estas tipologías constructivas: zonas intermedias de amortiguación térmica, orientación según trayectorias del sol, redireccionamiento de vientos, y captación de agua y energía.

Este plan propone desarrollar un parque universitario diferenciando sectores públicos de esparcimiento y privados de estudio. Donde cada área ligada a una disciplina de investigación tendrá su propio patio de estudio vinculado con símbolos desde el arte que recordaran la vida de algún personaje ilustre de la región. La comunicación entre patios de diferentes unidades académicas se articula mediante áreas de uso público. (ver figura 8).

Una consideración importante para favorecer el uso peatonal es restringir el uso del automóvil, dejando los estacionamientos en el perímetro y estimular el uso de las ciclovías.

Un espacio hito de esta propuesta es el foro localizado en el acceso al conjunto, reflejo de la voluntad por integrar la vida universitaria con el barrio y la ciudad. 


\section{Plan maestro "Parque botánico"}

Este master plan propone la consolidación de un gran parque, que favorezca la conexión entre la investigación académica y la naturaleza. Y simultáneamente permita aportar a la ciudad de Chillan un área verde que ayude en mejorar la grave deficiencia que presenta en este ámbito.
El parque está diseñado en base a una secuencia de recorridos aparentemente desordenados, pero que obedecen a entregar la noción de un libre caminar público sin interferir con la investigación y la educación. El manejo del agua se transforma en un elemento paisajístico que acompaña un paseo refrescante en verano, como separar y controlar el ingreso al área de investigación y experimentación.
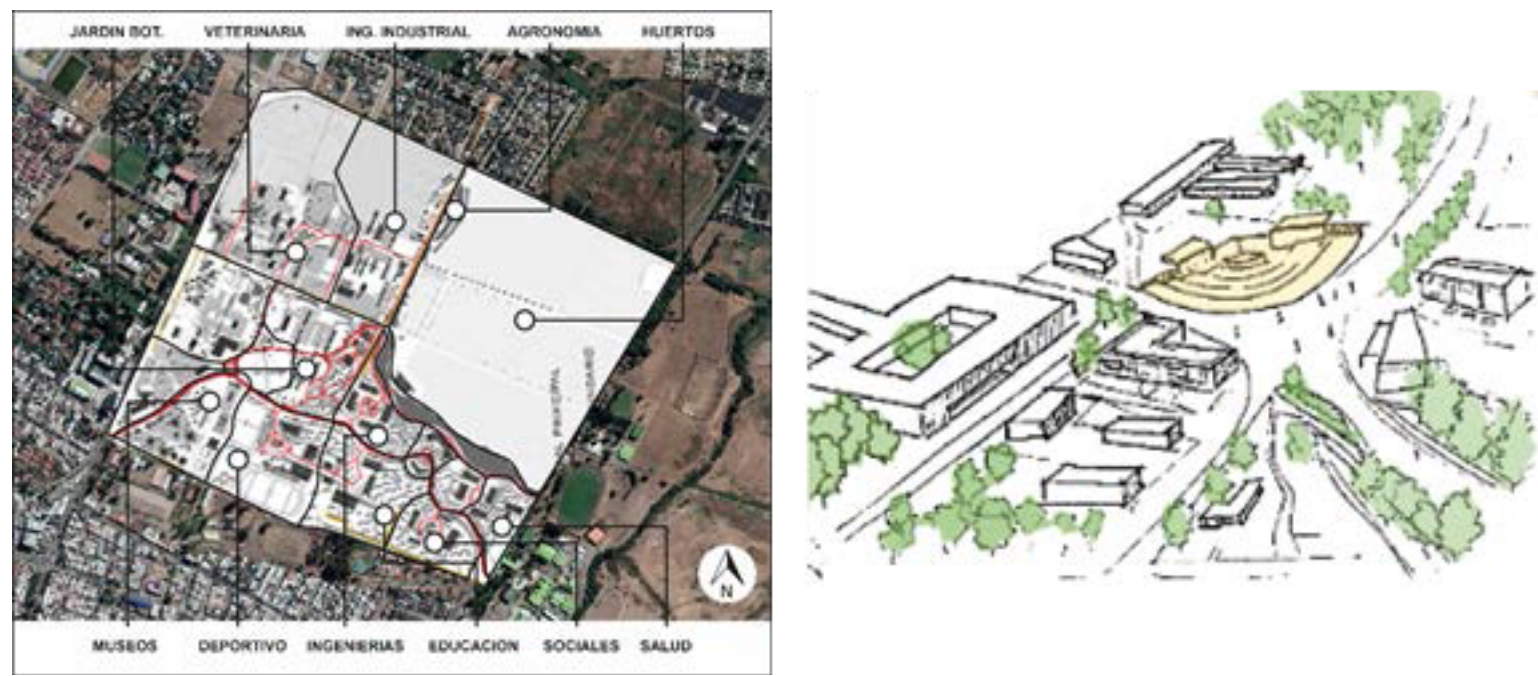

Figura 9. Masterplan Parque histórico universitario. Fuente: Francisco Fuentemavida, Manuel González, Gabriela Salgado y Esteban Vásquez.

Los tres circuitos principales, articulan el conjunto con su entorno inmediato en la escala del barrio, reconociendo la expansión residencial y la existencia del campus de la Universidad del Bio Bio. Estos circuitos confinan de forma imperceptible los diferentes núcleos relacionados con las diferentes carreras que se imparten dentro de este Parque Botánico.

\section{Plan maestro "Campus sustentable"}

A escala local y regional, el plan maestro ofrece una visión de campus que está en armonía con su medio físico y resguarda su entorno natural, potenciado mediante la preservación de biodiversidad y la investigación científica.

En la escala de la ciudad propone plazas articuladoras con el entorno urbano. La principal ubicada al surponiente, consta de un foro cívico, y un mercado agrícola que comercializa productos del campus, así como de productores del área rururbana, potenciando la economía local. (ver figura 10).

En la escala del barrio propone redes peatonales que cruzan el campus e interconectan barrios adyacentes. Estos recorridos acompañados de un sistema de áreas verdes resguardados por distintas edificaciones al interior del campus. 

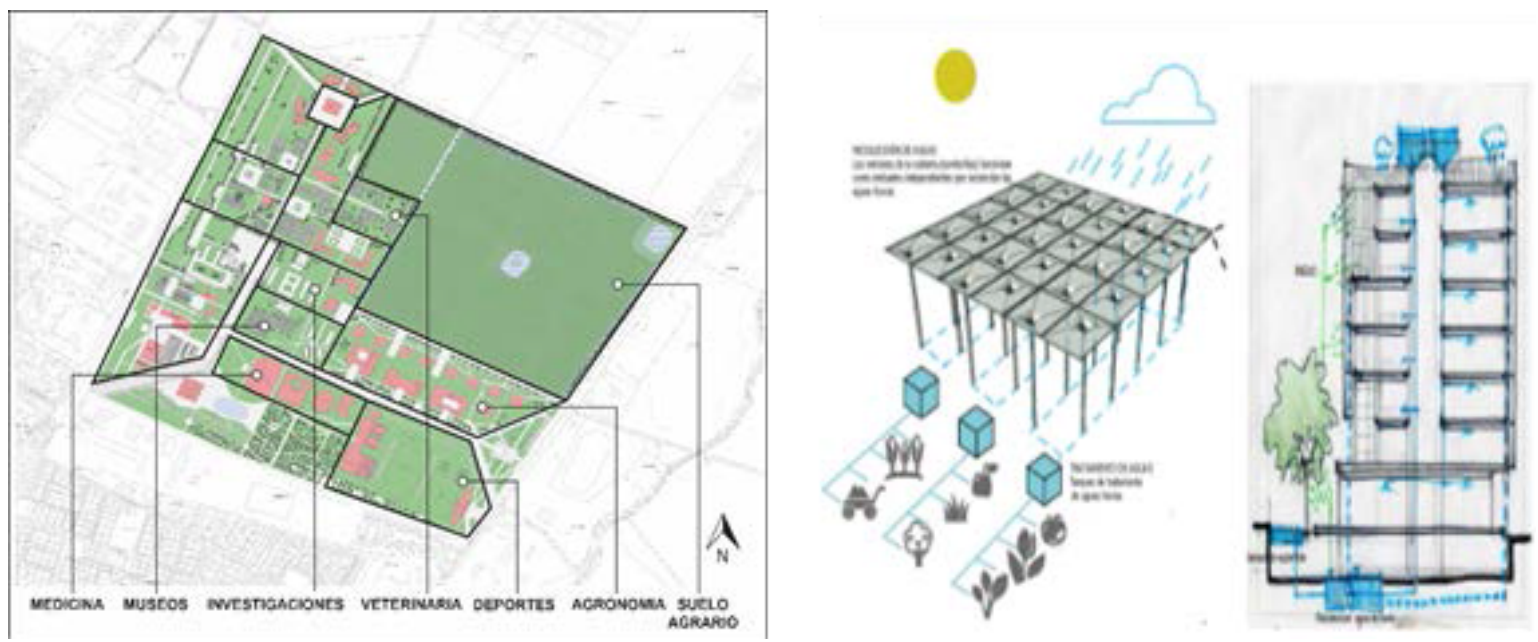

Figura 10. Masterplan Campus Sustentable. Fuente: Daniela Miranda, Álvaro Pérez, Tamara Yaritza y María Vergara.

Estas edificaciones constan de cubiertas verdes para la captación de aguas lluvias, utilizables para el regadío de cultivos y áreas verdes. Sistema de paneles fotovoltaicos para la autonomía energética, e innovación a través del cultivo en huertas verticales. Complementariamente, se crea un sistema de recuperación de los desechos provocados por la Universidad como por los barrios adyacentes. Estos se tratan y reutilizan para nutrir los suelos de vocación agrícola, potenciando un metabolismo circular dentro del campus.

\section{CONCLUSIONES}

\section{Potencialidades del campus para el desarrollo futuro de Chillán}

La historia del campus Chillán deja en evidencia su vocación agrícola, que ha estado articulada desde los inicios con las potencialidades de desarrollo de la ciudad y el desarrollo local de su región (Dalton et al., 2018; Rossi y Goglio, 2020). No obstante, las dinámicas de crecimiento urbano contemporáneo están amenazando las potencialidades agrícolas de la zona y segregando socialmente a sus habitantes y espacios de encuentro (Anabalón, 2006; Soto y Escobar, 2016).
La carencia de diversidad y planeamiento urbano dificulta la conexión y dependencia del Campus con el centro urbano de Chillán Nuevo. Simultáneamente, se observa una ausencia de equipamientos de cultura y áreas verdes en los segregados barrios en las inmediaciones del campus.

Todo lo anterior respalda la posibilidad de concebir el Campus Chillán como una nueva centralidad para la ciudad, rescatando los principios fundacionales del masterplan de Brunner y Duhart en Concepción y generando un sello institucional donde todos los campus de la Universidad de Concepción ofrezcan espacios comunes y colectivos para el uso de la ciudad (Fuentes, 2007), contribuyendo a la identidad regional y a la mejora en la calidad de vida y economía local (Dalton et al., 2018 ; Rossi y Goglio, 20201.

Bajo esta lógica, las alternativas de diseño que apuntan a densificar los bordes del campus y resguardar las áreas verdes y de cultivo a su interior lanillo construido interior verdel emergen como excelentes estrategias para aprovechar las plusvalías del suelo, mejorar la conexión y dotación de equipamientos y comercio entre barrios y, 
simultáneamente, resguardar el "laboratorio vivo" más importante del país (Soto y Escobar, 2016).

Además, los nodos articuladores campusciudad en los vértices suroriente y surponiente emergen como estrategias reiterativas en los planes maestros, que permiten disponer de zonas de uso público para la ciudad, y de articulación con la Universidad del Bío-Bío.

Finalmente, un buen aprovechamiento de las tipologías constructivas permitiría rescatar los principios de diseño de las haciendas de campo de la zona central, incorporando nuevas tecnologías para el aprovechamiento de aguas lluvias, desechos orgánicos y autonomía energética, desarrollando medidas apropiadas para rescatar las identidades locales y, simultáneamente, ponerse a la vanguardia nacional mediante el desarrollo de un campus sustentable.

En conclusión, proyectar el campus como un nuevo núcleo urbano, incorporando las bondades de la infraestructura verde, soberanía alimentaria y autonomía energética, son enfoques pioneros que permitirían posicionar al campus Chillán como un referente a nivel nacional e internacional, así como contribuir a robustecer el sello institucional y mejorar la calidad de vida y sostenibilidad urbana de la nueva capital de la Región de Nuble. 


\section{REFERENCIAS BIBLIOGRÁFICAS}

Alcántara, E. (2010). Análisis de la movilidad urbana. Espacio, medio ambiente y equidad. Bogotá: CAF. Retrieved from http://scioteca. caf.com/handle/123456789/414

Anabalón, N. (2006). Intercomunal Chillán, una ciudad morfológica y socialmente fragmentada en busca del desarrollo sustentable. Urbano, 9(13), 92-94. Recuperado de http://revistas. ubiobio.cl/index.php/RU/article/view/482

Briones, F., Hernández, C. y Mancilla, B. (2019). La escuela práctica de agricultura de Chillán y su importancia para la agricultura regional, 1881 hasta la década de 1930. Tiempo y Espacio, (40), 54-62. Recuperado de http://revistas.ubiobio.cl/index.php/TYE/ article/view/3915

Cáceres, C. y Ahumada, G. (2018). Evaluación de brechas de equipamiento urbano entre barrios de Viña del Mar, Chile: una metodología para la identificación de desiertos urbanos. Investigación Geográfica, (97), 1-22 . http://dx.doi.org/10.14350/ rig. 59615

Coss Lanz, A. (2010). Revisión Histórica del paisajismo de la Ciudad Universitaria de Caracas, patrimonio común universal. Apuntes, 22(2), 156-171. http://www. scielo.org.co/scielo. php?.pid=S 1657 $97632009000200006 \&$ script=sci_ arttext\&thng=en

Dalton, L., Hairasouliha, A. y Riggs, W. (2018). State of the Art in Planning for College and University Campuses: Site Planning and Beyond. Journal of American Planning Association, 84(2), 145-161. https://doi.org / 10.1080/01944363.2018.1435300

Fuentes, P. (2007). Campus universitarios en Chile: nuevas formas análogas a la ciudad tradicional. Atenea, (496), 117 $144 . \quad$ http://dx.doi.org/10.4067/ s071804622007000200008
García-Huidobro, A. y Maragaño, A. (2010). La vertebración territorial en regiones de alta especialización: Valle central de Chile. Alcances para el desarrollo de zonas rezagadas en torno a los recursos naturales. EURE, 36(107), 49-65. https://doi.org/10.4067/SO25071612010000100003

Gonzalez, S. y Olave, D. (1996). Áreas Verdes Urbanas en la Ciudad de Chillán. Chile. Tiempo y Espacio, (6), 75-83. http:// revistas.ubiobio.cl/index.php/TYE/article/ view/1592

Lizárraga, C. (2006). Movilidad urbana sostenible: un reto para las ciudades del siglo XXI. Economía, Sociedad y Territorio, VI(22), 283-32 1. https://biblat.unam.mx/hevila/ Economiasociedadyterritorio/2006/volb/ no22/1.pdf

Llop, J., Iglesias, B., Vargas, R. y Blanc, F. (2019). Las ciudades intermedias: concepto y dimensiones. Ciudades, (22), 22-43. https://doi.org/10.24197/ ciudades. 22.2019.23-43

Rossi, F. y Goglio, V. (2020). Studies in Higher Education Satellite university campuses and economic development in peripheral regions peripheral regions. Studies in Higher Education, 45(1), 34-54. https://doi.org/10.1080/03 075079.2018 .1506917

Rueda, S. (2012). El Urbanismo Ecológico. Barcelona: Agencia de Ecología Urbana. https: / / www.researchgate.net/profile/ Salvador_Rueda/publication/267827504_ E L_URBA A ISMO_ECOLOGICO/ links/564cadc908ae352ab55al4ef.pdf

Soto, M. y Escobar, A. (2016). Puesta en Valor del Patrimonio Agrícola en Ciudades Intermedias, caso Chillán. Revista de Urbanismo, (35), 145-166. https://doi. org/10.5354/0717-5051.2016.40050

Universidad de Concepción (2020). Historia Campus Chillán. Recuperado de http:// chillan.udec.cl/index.php/historia/ 